\title{
Cortical processing of facial tactile stimuli in temporomandibular disorder as revealed by magnetoencephalography
}

\author{
Aurelio A. Alonso - Ioannis G. Koutlas • \\ Arthur C. Leuthold · Scott M. Lewis · \\ Apostolos P. Georgopoulos
}

Received: 2 March 2010 / Accepted: 4 May 2010 / Published online: 26 May 2010

(C) US Government 2010

\begin{abstract}
We used magnetoencephalography (MEG) to investigate the cortical processing of an innocuous facial tactile stimulus in healthy subjects and in a group of subjects suffering from chronic temporomandibular disorder (TMD). Equivalent current dipoles (ECDs) were extracted for a time period of $1 \mathrm{~s}$ following stimulus application, and their location, duration and onset time determined. The counts of ECDs extracted did not differ significantly
\end{abstract}

A. A. Alonso - I. G. Koutlas · A. C. Leuthold - S. M. Lewis ·

A. P. Georgopoulos

Brain Sciences Center, Veterans Affairs Medical Center,

Minneapolis, MN 55417, USA

\author{
A. A. Alonso · A. P. Georgopoulos \\ Graduate Program in Oral Biology, \\ University of Minnesota, Minneapolis, \\ MN 55455, USA
}

I. G. Koutlas $(\bowtie)$

Department of Diagnostic and Biological Sciences,

School of Dentistry, University of Minnesota,

Room 16-108A Moos Tower, 515 Delaware Street S.E.,

Minneapolis, MN 55455, USA

e-mail: kout1001@umn.edu

A. C. Leuthold · A. P. Georgopoulos

Department of Neuroscience, University of Minnesota Medical

School, Minneapolis, MN 55455, USA

S. M. Lewis · A. P. Georgopoulos

Department of Neurology, University of Minnesota Medical

School, Minneapolis, MN 55455, USA

A. P. Georgopoulos

Department of Psychiatry, University of Minnesota Medical

School, Minneapolis, MN 55455, USA

\section{A. P. Georgopoulos}

Center for Cognitive Sciences, University of Minnesota,

Minneapolis, MN 55455, USA between the two groups. In contrast, we found statistically significant differences in ECD duration and onset time. Specifically, ECD duration was longer in the TMD group in the precentral gyrus, and ECD onset time was earlier in the parietal operculum. In addition, we found differences in the internal organization and clustering of the brain areas involved indicating a less tight association and a less coordinated stimulus information processing in the TMD group. Altogether, these results show that an innocuous facial tactile stimulus is differently processed in the brain of TMD subjects, when compared to controls, reflecting altered brain mechanisms due to chronic pain.

Keywords Magnetoencephalography ·

Temporomandibular disorder · Tactile stimulation .

Equivalent current dipole $\cdot$ Orofacial pain

\section{Introduction}

Magnetoencephalography studies have been carried out to study the spatial and temporal characteristics of brain responses following the application of stimuli of different modalities on body structures innervated by the trigeminal nerve, including electrical (Bessho et al. 2007; Nagamatsu et al. 2001; Nakahara et al. 2004; Nakasato et al. 2001; Sakamoto et al. 2008a, b) and tactile stimulation (Hoshiyama et al. 1995; Nakamura et al. 1998; Nevalainen et al. 2006; Nguyen et al. 2004, 2005; Yang et al. 1993). Electrical stimulation of three different areas of the hard palate elicited ECDs that were localized along the central sulcus and were oriented along an antero-inferior axis (Bessho et al. 2007). Nagamatsu et al. (2001) obtained similar results after electrical stimulation of the lower lip. In this case, a short-latency (14.6 ms) ECD was also localized in 
the central sulcus, along an antero-superior axis. In tactile stimulation studies, facial skin areas were stimulated by airdriven membrane in six regions of the face, and the resulted ECDs were localized in the first somatosensory cortex (SI) (Nguyen et al. 2004). Subsequently, three facial skin areas were stimulated by an air-driven membrane in order to map the facial skin representation in the second somatosensory cortex (SII). The results obtained showed that facial skin and lip are also represented in SII, although the representation of facial skin in SII is small (Nguyen et al. 2005). The representation of the facial skin in SI is located between the thumb and lip (Nguyen et al. 2004). Finally, a quantitative sensory test (QST) was performed in subjects with idiopathic facial pain who showed abnormal dishabituation of the R2 component of the blink reflex. It was found that the QST was not altered in these patients. In addition, the distance between the cortical representations of the lip and index finger did not differ significantly between patients and controls, suggesting that idiopathic facial pain is maintained by mechanisms which may not involve somatosensory processing of stimuli from the painful skin (Lang et al. 2005).

Several functional neuroimaging studies have been performed to investigate cortical function in patients suffering from orofacial pain, including positron emission tomography (PET) (Derbyshire et al. 1994; Hagelberg et al. 2003; Kupers et al. 2000, 2004) and functional magnetic resonance imaging (fMRI) (Albuquerque et al. 2006; Blatow et al. 2009; Borsook et al. 2003; DaSilva et al. 2007, 2008; de Leeuw et al. 2005, 2006; Jantsch et al. 2005; Jiang et al. 2006; Morelli et al. 2008; Nash et al. 2007). It has been found that SI and SII participate in the perception of sensory features of pain, together with other cortical areas (Apkarian et al. 2005; Bushnell et al. 1999).

In a PET study, Derbyshire et al. (1994) measured regional the cerebral blood flow (rCBF) response in patients suffering from chronic atypical facial pain and in healthy control subjects following delivery of innocuous (non-painful) and painful thermal stimuli. Both groups showed significant differences in $\mathrm{rCBF}$ response to painful versus non-painful heat stimuli in several brain areas, including the thalamus, anterior cingulate cortex (area 24), lentiform nucleus, insula and prefrontal cortex. Increased $\mathrm{rCBF}$ was found in the anterior cingulate cortex and decreased in the prefrontal cortex in the atypical facial pain group, documenting the importance of the cingulate cortex and its reciprocal connections with the prefrontal cortex in processing of pain (Derbyshire et al. 1994).

Kupers et al. (2000) measured rCBF in patients suffering from chronic orofacial pain. They found significantly increased $\mathrm{rCBF}$ in the prefrontal cortex, anterior cingulate cortex, anterior insular cortex, hypothalamus and periaqueductal gray matter. In contrast, $\mathrm{rCBF}$ was decreased in the substantia nigra, red nucleus and anterior pulvinar. Kupers et al. (2004) also investigated the cerebral activation patterns elicited by experimental jaw-muscle pain induced by injections of 5\% hypertonic saline into the right masseter muscle. This led to rCBF increase in the dorsal-posterior insula (bilaterally), anterior cingulate and prefrontal cortices, right posterior parietal cortices, brainstem, and cerebellum. No changes were found in SI or SII. In contrast, stimulation by von Frey hairs increased rCBF in the contralateral face representation in the SI (Kupers et al. 2004).

Several fMRI studies have attempted to evaluate brain function in patients with trigeminal pain. Borsook et al. (2003) assessed the activation of the trigeminal ganglion using innocuous mechanical (brush) stimuli and noxious thermal stimuli applied to the face. Changes in BOLD signal were observed in the ipsilateral ganglion with either stimulus type; specifically, the signal decreased after brush stimuli and increased after noxious stimuli. In another fMRI study, de Leeuw et al. (2006) found that painfully hot stimulation of the skin overlying the left masseter muscle activated discrete brain regions within the insula, cingulate gyrus, thalamus, inferior parietal lobe, postcentral gyrus, right middle and inferior frontal gyri, cuneus, precuneus, and precentral gyrus. This pattern of activation resembles that involved in pain processing of painful peripheral stimuli. (de Leeuw et al. 2006). In contrast, electrically induced tooth pain activated SI, SII and the insula bilaterally (Jantsch et al. 2005), a distinctly different activation pattern from that elicited by painful stimulation of the hand.

Nash et al. (2007) investigated the cortical and subcortical patterns of activation elicited by experimentally induced jaw-muscle pain in the right masseter muscle. The results revealed significant activation in the spinal trigeminal nucleus (the SI relay nucleus serving the orofacial region) as well as in the left (contralateral) SI and posterior insular cortex, and the right (ipsilateral) anterior insular cortex. In contrast, the perigenual cingulate cortex showed significantly decreased signal intensity (Nash et al. 2007).

Blatow et al. (2009) tested whether pain is associated with an alteration in somatosensory function in patients with trigeminal neuralgia (TN) using fMRI. A tactile non-painful stimulus was delivered in the lips and fingers. There were three groups, as follows. The first group consisted of patients with TN, the second group consisted of patients with TN relieved after surgery, and the third group consisted of healthy control subjects. It was found that the activation of SI and SII did not differ significantly between operated and non-operated subjects. On the other hand, patients with TN showed a significant reduction in SI and SII activation, when compared to controls, and these differences were more prominent during stimulation of the fingers. With respect to lip stimulation, SI and SII showed a reduced activation on the contralateral but not on the ipsilateral side to the 
stimulus application. This reduced activation probably reflects reduced neural processing, due perhaps to long-term adaptation to painful stimuli (Blatow et al. 2009).

Jiang et al. (2006) compared the brain activation patterns after evoked clenching, among three groups of subjects, namely patients with atypical facial pain, patients who had occlusal treatment for synovitis pain of temporomandibular disorder (TMD), and normal subjects. They found that different regions were activated between the two patient groups. Specifically, the atypical facial pain activated the thalamus and anterior cingulate cortex, whereas the synovitis pain of TMD activated SI and prefrontal cortex. It was concluded that the pain network is different between the two pain groups and that the pain network is more sensitive in patients with atypical pain (Jiang et al. 2006).

Albuquerque et al. (2006) studied patients with burning mouth disorder (BMD) and normal controls after thermal stimulation of the right masseter. The BMD group showed greater fractional signal changes in the right anterior cingulate cortex (areas 32/24) and the precuneus (bilaterally). The control group showed larger signal changes in the thalamus bilaterally, the right middle frontal gyrus, the right precentral gyrus, the left lingual gyrus, and the cerebellum. In general, patients with BMD showed brain activation patterns similar to those observed in patients with other neuropathic pain conditions than those observed in control subjects. These results suggest that brain hypoactivity may be an important feature in the pathophysiology of BMD (Albuquerque et al. 2006). The same authors reviewed the supraspinal mechanisms in pain derived from neuroimaging studies and concluded that chronic orofacial pain conditions may be related to a dysfunctional brain network and may involve a deficient descending inhibitory system. The somatosensory cortices, anterior cingulate cortex, thalamus, and prefrontal cortex may play a vital role in the pathophysiology of chronic pain and should be the main focus of future neuroimaging studies in chronic pain patients (de Leeuw et al. 2005).

In patients with chronic trigeminal neuropathic pain (right maxillary), changes in cortical thickness were found, including both cortical thickening and thinning in sensorimotor regions (SI, SII, and precentral gyrus), and predominantly thinning in other regions (dorsolateral prefrontal cortex, frontal cortex, anterior insula, and cingulate cortex). The patterns of alteration in cortical thickness suggest a dynamic functionally driven plasticity of the brain. Interestingly, these structural changes, which correlated with the pain duration, age-at-onset, pain intensity, and cortical activity were frequently co-localized and correlated with functional allodynic SI and SII activations documented by fMRI, and they may be specific targets for evaluating therapeutic interventions (DaSilva et al. 2008). Finally, investigation of the cerebral activation in cluster headache before, during and after an attack, showed significant hypothalamic activation of the hypothalamus ipsilateral to the pain side (DaSilva et al. 2007; Morelli et al. 2008).

In summary, several studies using diverse methodologies have found differential involvement of several brain areas following trigeminal stimulation, and also differences in brain activation between healthy people and patients suffering from orofacial pain. In this study, we used magnetoencephalography (MEG; Hamalainen et al. 1993) to investigate the dynamic neural mechanisms underlying the processing of an innocuous facial tactile stimulus in healthy subjects and in patients suffering from chronic TMD pain group. We sought to assess differences between the two groups with respect to the strength, localization, and dynamic aspects of cortical activation, as well as to evaluate the grouping patterns of the areas involved and how they may change in TMD. For that purpose, we employed the multivariate analyses of multidimensional scaling (MDS) and hierarchical tree clustering (HTC) which have been used with success in previous neurophysiological (Merchant et al. 2003) and fMRI studies (Tagaris et al. 1997, 1998; Tzagarakis et al. 2009).

\section{Materials and methods}

\section{Subjects}

Eighteen subjects were studied as paid volunteers. Ten subjects were healthy controls without pain (7 women and 3 men, age (mean $\pm \mathrm{SD}$ ) $30.8 \pm 11.1$ years) whereas eight were suffering from chronic TMD (7 women and 1 man, $37.5 \pm 11.6$ years), according to the Research Diagnostic Criteria (RDC) (Dworkin and LeResche 1992). The study protocol was approved by the appropriate institutional review boards, and informed consent was obtained from all subjects before the study according to the Declaration of Helsinki.

\section{Stimulus delivery}

Subjects rested in a supine position with their eyes open. A circular elastic membrane, $1 \mathrm{~cm}$ in diameter, was attached to each side of the face on the top of the masseter muscle (Fig. 1). The motion of the membrane was triggered by a computer-generated air pressure stimulus of $17 \mathrm{lb}$ per square inch (psi). Two hundred stimuli of $50 \mathrm{~ms}$ duration each were delivered with a pseurandom interstimulus interval of 1-2 s. There was a delay of $41 \mathrm{~ms}$ from the onset of the trigger to the beginning of the motion of the membrane (Biermann et al. 1998).

\section{Data collection}

Magnetoencephalography data were collected using a 248-channel axial gradiometer system (Magnes 3600WH; 

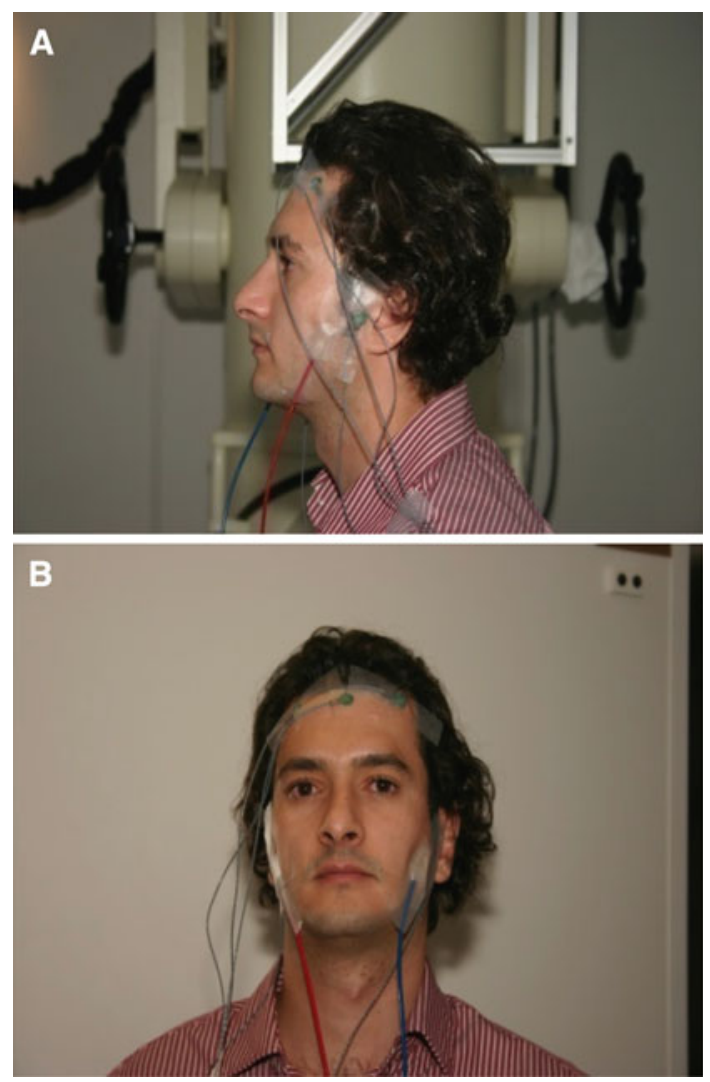

Fig. 1 Location of the air puff stimulators above the skin of the masseter muscle. a left side; $\mathbf{b}$ both sides

4D-Neuroimaging, San Diego, USA). The cryogenic helmet-shaped dewar of the MEG was located within an electromagnetically shielded room to reduce noise. Data $(0.1-400 \mathrm{~Hz})$ were collected at $1,017.25 \mathrm{~Hz}$. For anatomical source localization, T1-weighted gradient-echo images of structural MRI for each subject were acquired.

\section{Data preprocessing and dipole extraction}

The acquired time-series data were corrected for the cardiac artifact using event synchronous subtraction (Leuthold 2003). The integrated BESA (version 5.06, MEGIS Software $\mathrm{GmbH}$, Gräfelfing, Germany) and Brain Voyager (Electrical Geodesics, Inc, Eugene, OR, USA) package was used for single ECD extraction (Uutela et al. 1999; Wolters et al. 1999). This method derives dipolar current sources to approximate the flow of electrical current in a small brain area. Periods with noisy sensors and/or eye blink artifacts were detected and eliminated from further analysis using visual inspection and the BESA artifact rejection tool. Epochs were defined from -100 to $1,000 \mathrm{~ms}$ from stimulus onset and averaged aligned to stimulus onset. The averaged file was band-pass filtered (high-pass: $1 \mathrm{~Hz}$ second order; low-pass: $44 \mathrm{~Hz}$ fourth order; zero-phase). Possible ECD locations with dipolar distribution and correlated temporal evolution of pole intensity were then determined from derived flux maps of sensor waveforms by visual inspection for a period of $1,000 \mathrm{~ms}$ following stimulus onset. Only sensors (minimum 12) relevant to these locations were used for further dipole analysis of the respective ECD (Langheim et al. 2006). For each subject, the Brain Voyager software above was used to create a $3 \mathrm{D}$ reconstruction of the brain according to the acquired MRI images. Alignment between the Talairach coordinates of BESA and BrainVoyager was based on fiducials and surface points digitized during acquisition (Langheim et al. 2006). Using the BESA algorithm for dipole source modeling, ECDs were determined for each of the flux maps and projected onto the 3D-MRI reconstructions of their cortical location (Langheim et al. 2006). Acceptance criteria for ECD included (a) goodness of fit (GOF) of $>90 \%$, (b) maximum amplitude of $25 \mathrm{nA}$, (c) location in the cortical gray matter, and (d) duration $\geq 10 \mathrm{~ms}$. In more detail, several steps were taken into account using BESA software to fit a dipole. First, after eliminating the artifacts and averaging the data, we used fluxmaps to locate the detection coil groups of interest and selected several periods or fit interval in a period up to $1,000 \mathrm{~ms}$. The fit interval was defined as the duration of the magnetic source. The onset/offset times of the magnetic source were chosen based on the fit period peak, when the magnetic source reached the maximum peak (before it starts to fade) and based on that, we subtracted $20 \mathrm{~ms}$ for the onset and added $20 \mathrm{~ms}$ for the offset for closer view. Several magnetic sources can be selected in a period of $1,000 \mathrm{~ms}$, but not all of them will necessarily become a dipole. We only took into account dipoles that had $>90 \%$ GOF, located in the cerebral cortex, and a minimum of 12 sensors. It can happen that $\sim 40 \mathrm{~ms}$ fit period is too wide to generate a $>90 \% \mathrm{GOF}$; in that case, we decreased the width of the fit period until it reached $>90 \%$ GOF.

The fitting was not constrained by anatomical location and/or time. However, we did use time in order to localize the magnetic dipolar source in the 3D mapping for MEG (BESA tool). The time was selected based on the magnetic source in a period up to $1,000 \mathrm{~ms}$. No a priori assumptions were made regarding the number of partially simultaneous dipolar, and we performed a single dipole model in BESA. We choose BESA over the 4D Neuroimaging software because of the visualization tools.

For waveforms with multiple peaks, individual peaks were analyzed separately. The time of occurrence of each peak and a selection of a subset of sensors allowed a more accurate characterization of each ECD by avoiding interference from other fields. The onset time of derived ECDs was adjusted to account for the transmission delay $(41 \mathrm{~ms})$ of the stimulus (air puff) through the tubes.

Finally, measures of source amplitude were not considered in this study, since multiple, uncontrollable factors may influence these values. For sensor level measurements, 
small differences between subjects in the orientation of a small patch of cortex can have large effects on the measured signal amplitude. Similarly, a fold in a small active area will result in partial cancellation of the signal. In source space, a small error in estimation of source depth can have a substantial effect on the estimate of source strength. Interpretation of an amplitude difference is also difficult. An increase in amplitude is often interpreted as increased effort, but substantial past effort (i.e. a well practiced task) often results in a reduction of source amplitude. We realize that there are a growing number of papers showing a significant difference in amplitude for a particular comparison, but the number is very, very small compared to the number of reports where such a comparison is possible. This is likely an indication of the difficulties mentioned earlier. We would be more comfortable considering source amplitude when evaluating interictal spikes or comparing the response to different stimulus types within the same subject.

\section{Assignment to brain areas}

The assignment of dipoles to a specific cortical area was based on Talairach coordinates (Tailarach and Tournoux 1988) and anatomical landmarks. The following abbreviations are used below for the 23 areas where ECDs were found to be localized in this study: ParOper (parietal operculum), PoCGy (postcentral gyrus), PoCSul (postcentral sulcus), TemOper (temporal operculum), STGy (superior temporal gyrus), Ins (insula), PrCGy (precentral gyrus) PrCSul (precentral sulcus), MTGy (middle temporal gyrus), AngGy (angular gyrus), SMGy (supramarginal gyrus), STSul (superior temporal sulcus), LinGy (lingual gyrus), SPL (superior parietal lobule), CSul (central sulcus), IFGy (inferior frontal gyrus), Pcun (precuneus), Cun (cuneus), LatSul (lateral sulcus), CingGy (cingulate gyrus), IntParSul (internal parietal sulcus), ITGy (inferior temporal gyrus), OccGy (occipital gyrus), SFGy (superior frontal gyrus).

\section{Time-frequency analysis}

Epochs were defined between -100 and $1,000 \mathrm{~ms}$ from stimulus onset. Time-frequency analysis was performed per subject, condition, and sensor using the BESA package above. A bandwidth from 4 to $400 \mathrm{~Hz}$ was analyzed (every $2 \mathrm{~Hz}$ ) for a period of $1,100 \mathrm{~ms}(-100$ to $1,000 \mathrm{~ms}$, every $25 \mathrm{~ms}$; the period of -100 to $0 \mathrm{~ms}$ was stimulus-free and served as the baseline). The analysis yielded normalized changes in power over the baseline at a given frequency and time bin. For statistical analysis, data were binned in 20 frequency bands, every $20 \mathrm{~Hz}$, and 40 time bins of $25 \mathrm{~ms}$ bin width. (The first frequency bin contained frequencies 4$20 \mathrm{~Hz}$.) This yielded a $20 \times 40$ rectangular data matrix for each subject and sensor.
Statistical analyses: general

Standard statistical analyses, including parametric and nonparametric tests, were performed on ECD attributes to evaluate differences between the two groups within specific brain areas. These attributes comprised ECD counts, duration, and onset time. ECD counts were square-root transformed prior to analyses to normalize their distribution and stabilize the variance (Snedecor and Cochran 1989).

Statistical analyses: multivariate

These analyses aimed to explore the interrelations among the areas activated. Two multivariate analyses were applied, namely multidimensional scaling (MDS) and hierarchical tree clustering (HTC) (Shepard 1980). For both analyses, the input data consisted of the square-rooted ECD counts in 23 cortical areas. The MDS analysis was performed using the ALSCAL procedure of the SPSS statistical package (version 15) with the following parameters: the dissimilarities calculated were Euclidean distances, the model was ordinal, the matrix was conditioned, and the solution was two-dimensional. The HTC analysis was performed using the CLUSTER procedure of the SPSS statistical package above with the following parameters: the dissimilarities were squared Euclidean distances, and the cluster method was between-groups linkage.

\section{Results}

ECD counts

A total of 324 ECDs were found in 23 different cortical areas in the 18 subjects studied (Fig. 2). An analysis of covariance (ANCOVA) of the overall counts with Group as the fixed factor and Gender and Age as covariates found no statistically significant difference between the two groups $(P=0.264, F$-test $)$; in addition, neither Gender nor Age had a significant effect $(P=0.829$ and 0.467 , respectively). A $t$ test on the dipole counts for each area separately did not reveal any statistically significant difference between the two groups.

\section{ECD duration}

An ANCOVA on the ECD duration across areas yielded a highly significant difference between the two groups $(P=0.001, \quad F$-test; mean \pm SEM ECD duration: control $=26.1 \pm 0.874 \mathrm{~ms}, \mathrm{TMD}=31.43 \pm 1.066 \mathrm{~ms})$; neither Gender nor Age had a significant effect $(P=0.439$ and 0.431 , respectively). A $t$-test on the ECD duration for each area was performed. Given that data from 23 areas were 
Fig. 2 Average number of dipoles in various brain in the two groups. There were no significant differences in the average number of dipoles between the two groups in anyone of these areas

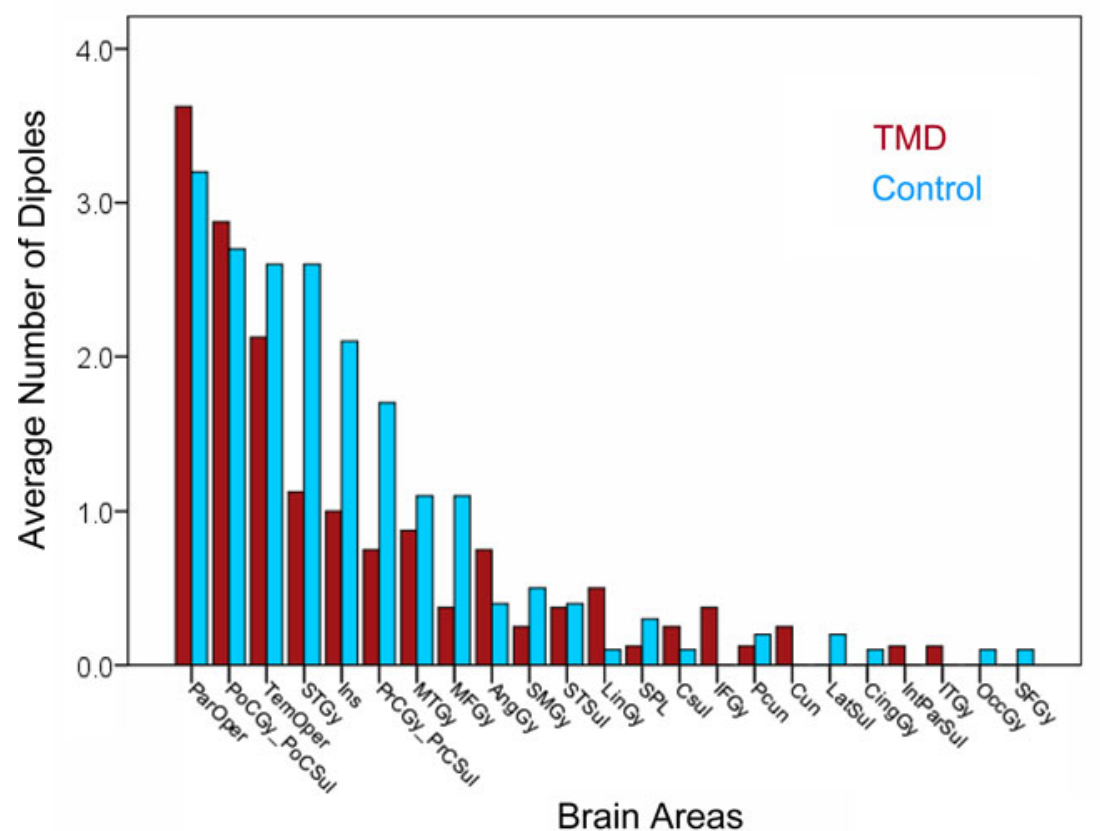

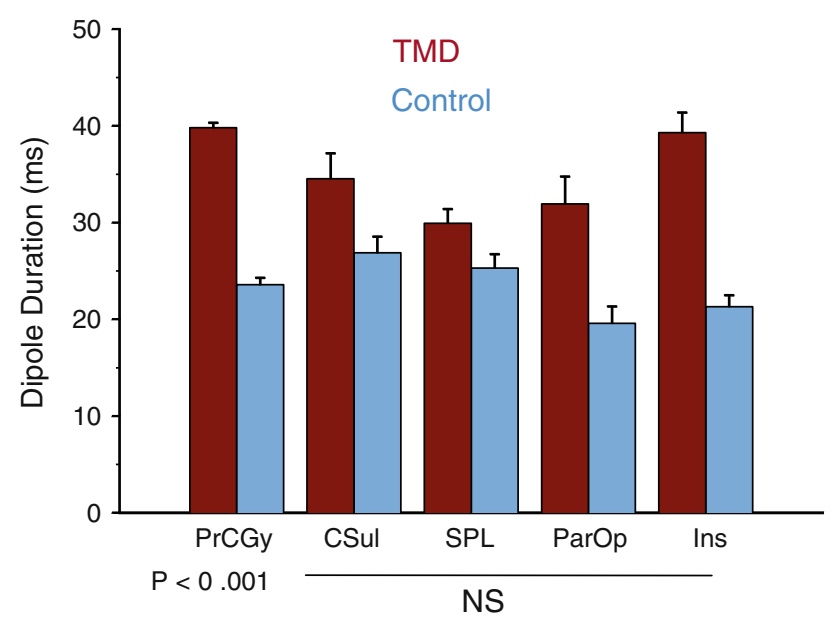

Fig. 3 ECD duration. ( $P$-value is from $t$-test, see text)

analyzed, the significance level was adjusted for multiple comparisons to $P<0.05 / 23$, i.e. $P<0.00217$. Longer duration in the TMD group was observed for five areas (Fig. 3) but was statistically significant only for PrCGy $(P=0.001)$.

\section{ECD onset time}

We used the log rank test to assess the statistical significance of differences in the onset time distributions between the two groups (Cox and Oakes 1984). Specifically, we focused on early onset times (up to $300 \mathrm{~ms}$ ) and analyzed data from the following areas with five or more ECDs per group: Ins (12 control, 5 TMD), ParOp (22 control, 21 TMD), PoCGy (15 control, 12 TMD), STGy (19 control, 6 TMD) and TempOp (18 control, 13 TMD). A statistically

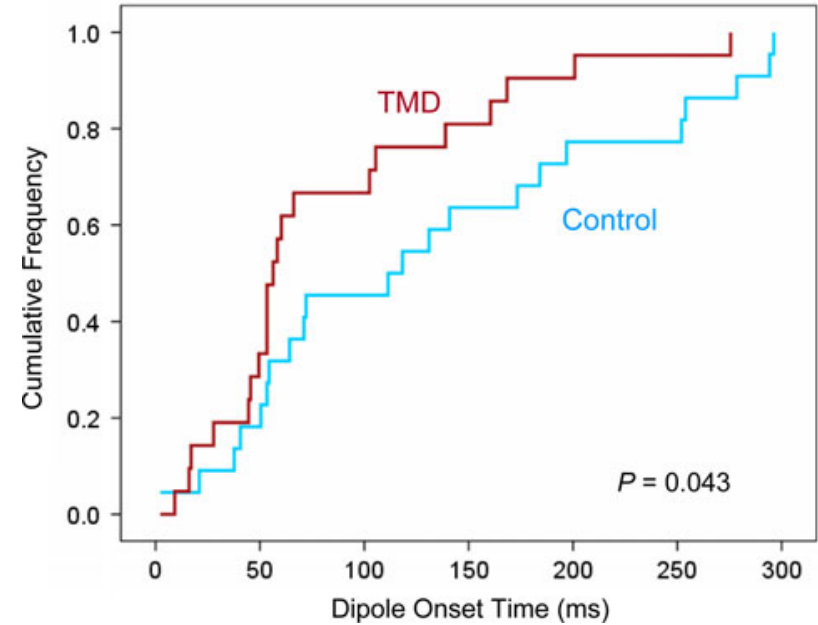

Fig. 4 Cumulative distributions of ECD onset times for the parietal operculum (SII). Notice that at $100 \mathrm{~ms}$, approximately $65 \%$ of the stimulus was already processed in the TMD pain group but only $45 \%$ in the control group

significant difference with earlier onset times in the TMD pain group was found only in ParOp $(P=0.043$; median control $=11.37 \mathrm{~ms}$, median TMD $=56.32 \mathrm{~ms})($ Fig. 4).

Multidimensional scaling

The analysis was successful, yielding an excellent goodness of it $\left(R^{2}=0.984\right)$. The area configuration plots obtained for the control and TMD groups are shown in Figs. 5 and 6 , respectively. It can be seen that areas closely involved with stimulus processing (PoCGy_PoCSul, ParOper, TempOper, Ins, STGy, enclosed within the blue ellipse in Fig. 5) are 
Fig. 5 Two-dimensional MDS plot of the 23 cortical areas for the control group (see text for details)
Fig. 6 MDS plot for the TMD group (see text for details)
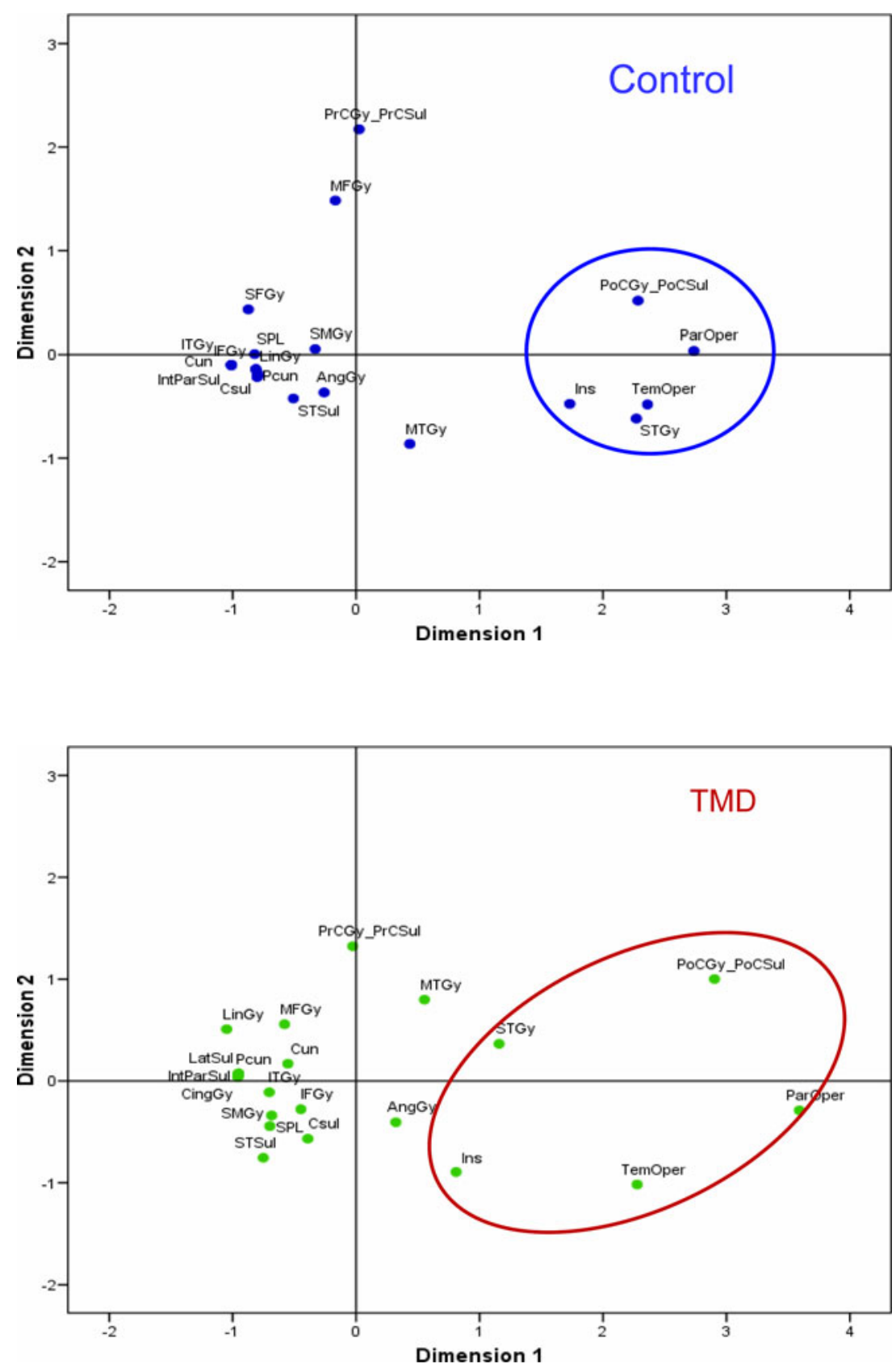

farther apart in the TMD group (within red ellipse in Fig. 6), indicating a more divergent, less coordinated information processing.

\section{Hierarchical tree clustering}

The trees (dendrograms) obtained for the control and TMD groups are shown in Figs. 7 and 8, respectively. It can be seen that the trees have similar structure in both groups, reflecting the same applied stimulus. Specifically, there are two main branches, an upper and a lower one that subdivides in two sub-branches. The former is separate whereas the latter contains partially overlapping smaller branches. Remarkably, and in spite of the overall formal similarity, these branches have different area composition in the two groups, most probably reflecting different stimulus processing. Specifically, a major difference is that area STGy has moved out of the lower branch (in the controls) to the upper branch (in the TMD group), whereas the other areas in that branch (PoCGy_PoCSul,ParOper, TempOper, Ins) remain the same. Thus, the lower branch contains one area less in the TMD group than in the control group. Finally, there is a reorganization of the areas in the various sub-branches of the upper branch. 
Fig. 7 Dendrogram of the 23 cortical areas with ECDs in the control group (see text for details)

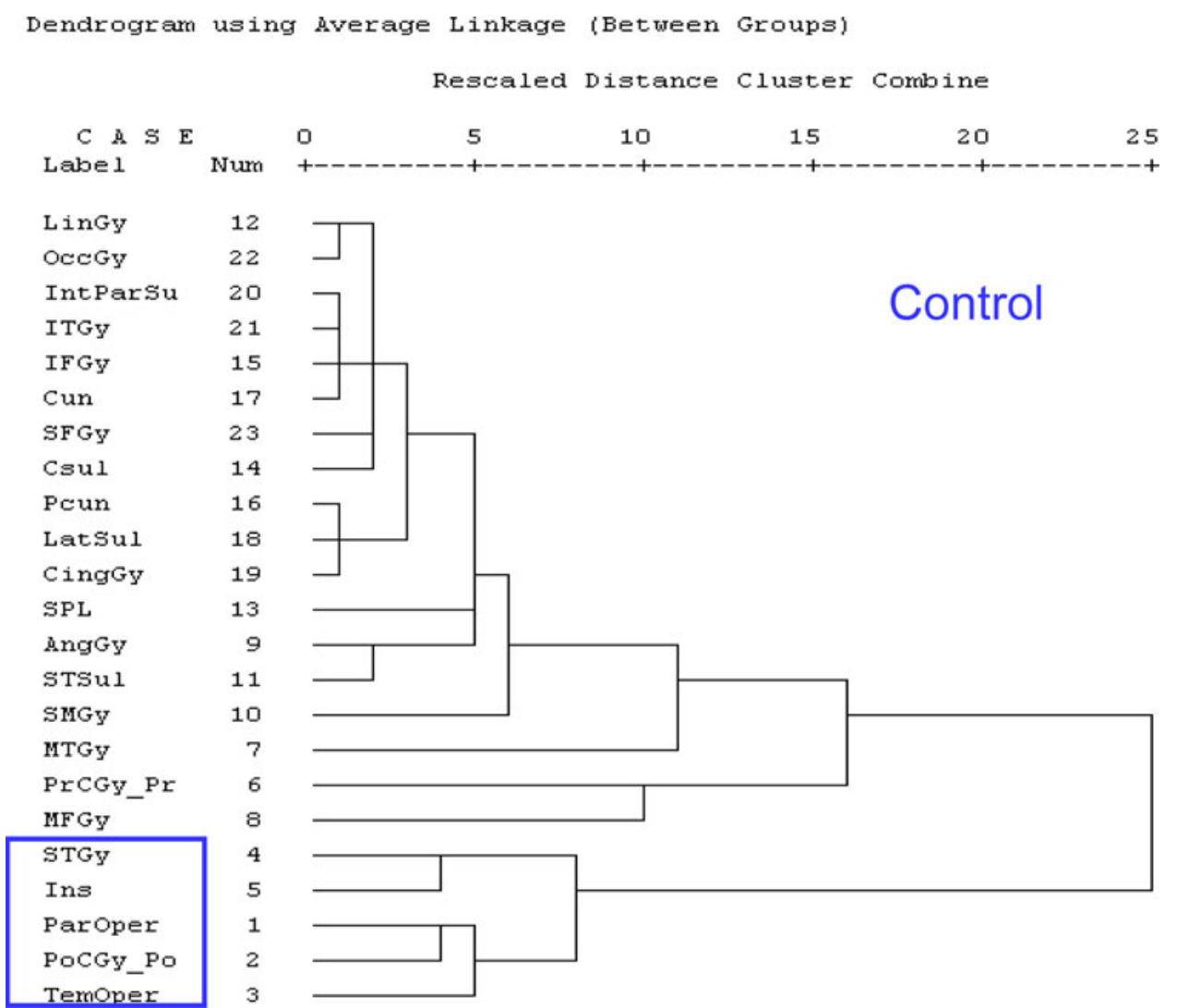

Time-frequency analysis

\section{Frequency effects}

For both groups, the average changes from baseline (across sensors and subjects) at different frequency bands (see "Methods" above) were negative for low frequencies (4-20 Hz) and positive for higher frequencies $(>20 \mathrm{~Hz})$. For this reason, we performed two ANOVAs, one for the 4-20 Hz frequency band and another for bands $>20 \mathrm{~Hz}$. We found that in both cases the power changes from baseline were stronger in the TMD pain versus the control group, i.e. more negative at $4-20 \mathrm{~Hz}$ and more positive at $>20 \mathrm{~Hz}$. Figure 9 illustrates the changes in the former $(P<0.001, t$-test $)$ and Fig. 10 the changes in the latter frequency bands. It can be seen in Fig. 10 that the TMD group showed consistently higher changes for the frequencies $21-160 \mathrm{~Hz}$. Statistically significant changes within particular frequency bins are indicated by a red asterisk $(P<0.001, t$-test $)$. The average changes within that range for each group are shown in Fig. $11(P<0.001, t$-test $)$. In contrast, the average changes from baseline in higher frequencies $(>160 \mathrm{~Hz})$ did not differ significantly between the two groups $(P=0.631, t$-test $)$.

\section{Time course}

The analysis above dealt with group differences in a frequency range collapsed across time bins. In this section, we examine and compare the changes from baseline between the two groups along time. We focus on the two frequency bands that showed statistically significant group effects, namely 4-20 and 21-160 Hz. Figure 12 plots the average (across the 248 sensors) time course of power changes for the $4-20 \mathrm{~Hz}$ frequency band. It can be seen that the TMD group showed consistently larger (negative) changes from baseline, extending up to $\sim 500 \mathrm{~ms}$ following stimulus onset. A repeated measures ANOVA showed a highly statistically significant effect of Group (between subjects factor), Time (within subjects factor) and Group $\times$ Time interaction $(P<0.001$ for all $F$-tests above). Such a pattern was evident consistently in all sensors.

Statistically significant effects were also found for the 21-160 Hz frequency band (Fig. 13); both the Group and Time main effects as well as the Group $\times$ Time interaction were highly statistically significant $(P<0.001$ for all 3 F-tests). Remarkably, the two curves diverged immediately following stimulus onset, with the TMD group manifesting consistently larger changes throughout the time course of $1 \mathrm{~s}$. 
Fig. 8 Dendrogram of the 23 cortical areas with ECDs in the TMD group (see text for details)
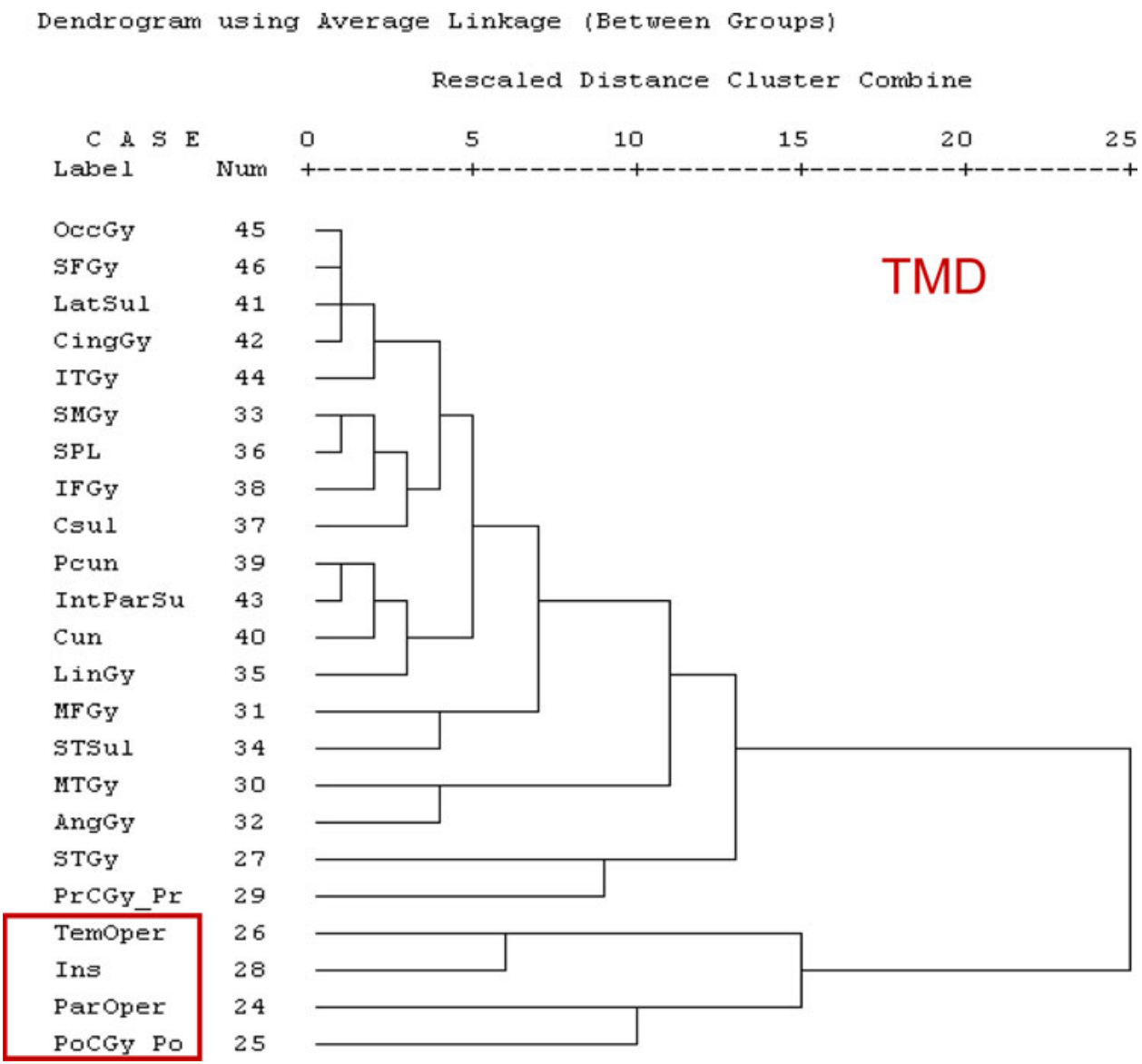

$4-20 \mathrm{~Hz}$

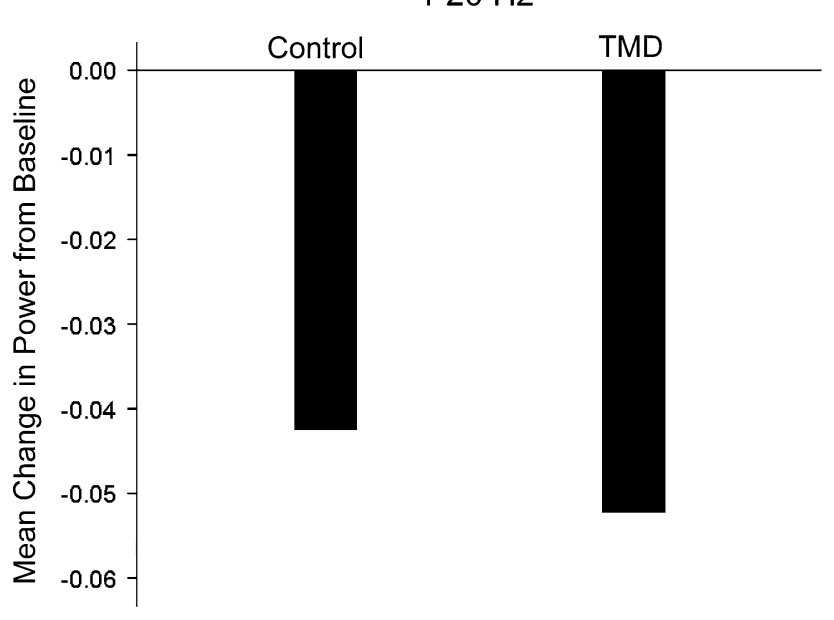

Fig. 9 Bar graphs show average changes in power from baseline in the control and TMD group for low frequencies $(4-20 \mathrm{~Hz}) . P<0.001, t$-test

\section{Discussion}

In this study, we compared control and TMD pain groups with respect to brain processing of a non-noxious tactile stimulus. We localized 324 dipoles in 23 different cortical

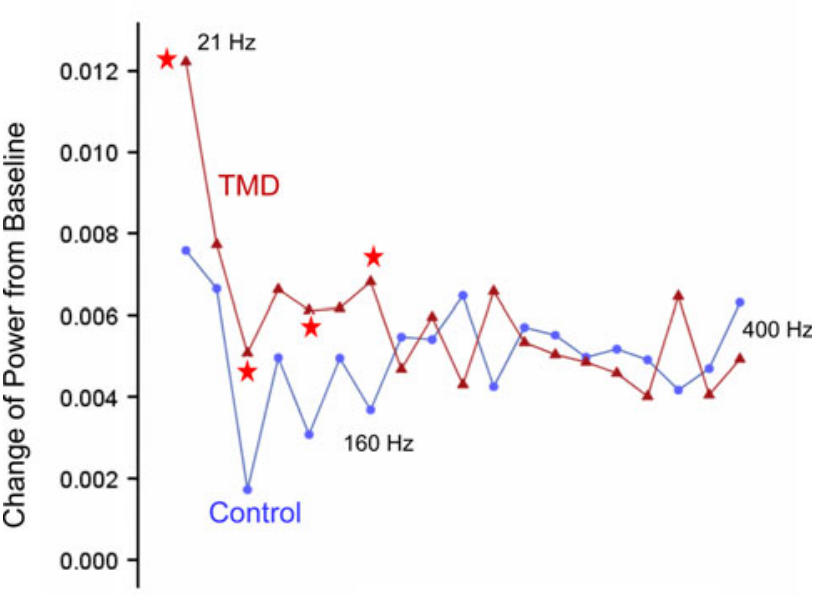

Fig. 10 Changes in power from baseline for different frequency bins above $20 \mathrm{~Hz}$. Asterisks denote statistically significant group differences $(P<0.001, t$-test $)$

areas in both groups. The majority of the dipoles were localized in SI and SII; the SII activation was bilateral, in accordance with the results of previous studies (Forss and Jousmaki 1998; Hari et al. 1993; Hoshiyama et al. 1996; Inui et al. 2003; Kakigi et al. 1995; Maeda et al. 1999). Interestingly, the ECD counts (i.e. the strength of activation) did not differ significantly in any area between the two 


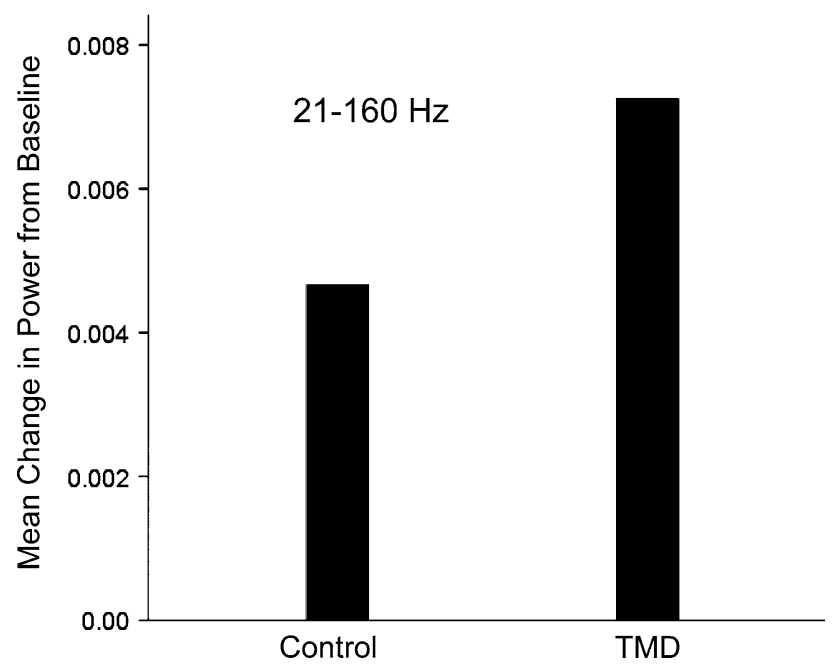

Fig. 11 Bar graphs show average changes in power from baseline in the control and TMD group for high frequencies $(21-160 \mathrm{~Hz})$. $P<0.001, t$-test

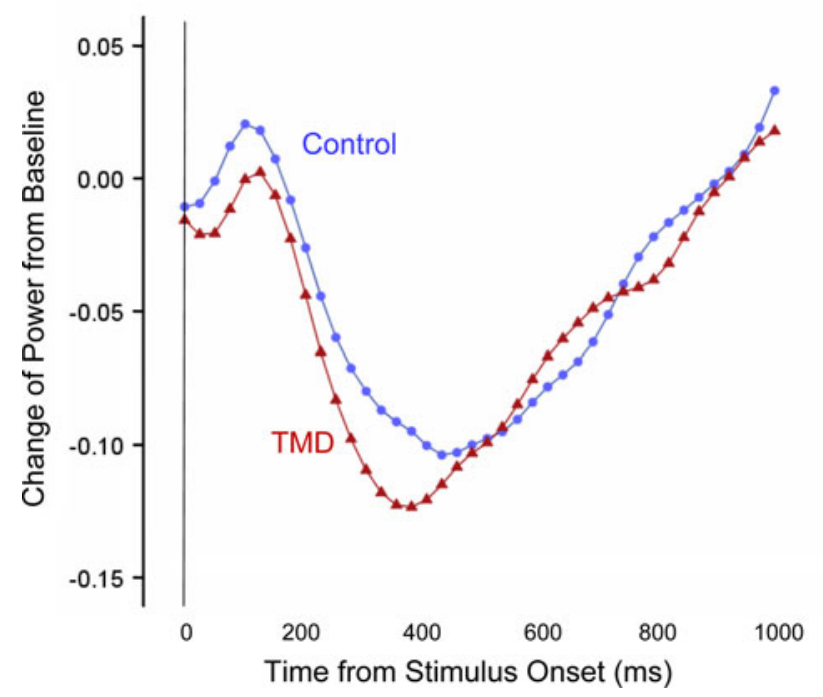

Fig. 12 Time course of power changes from baseline in the two groups for low frequencies $(4-20 \mathrm{~Hz})$

groups. In contrast, the most significant changes occurred in the temporal domain, in precentral, and parietal areas. Specifically, we found that the duration of the ECDs in five areas (PrCGy, CSul, SPL, ParOp, Ins) was statistically significantly longer in the TMD than the control group. We hypothesize that the TMD painful condition has altered the cortical mechanisms responsible for perceiving and processing non-noxious tactile stimuli, resulting in this persistent cortical activation. Such cortical plasticity has been demonstrated previously in SI in patients suffering from chronic pain after nerve injury (Jones 2000) and in patients with chronic trigeminal neuralgia (Jones 2000; Tronnier et al. 2001). Finally, we found that ECDs onset time was significantly shorter (i.e. activation appeared earlier) in SII

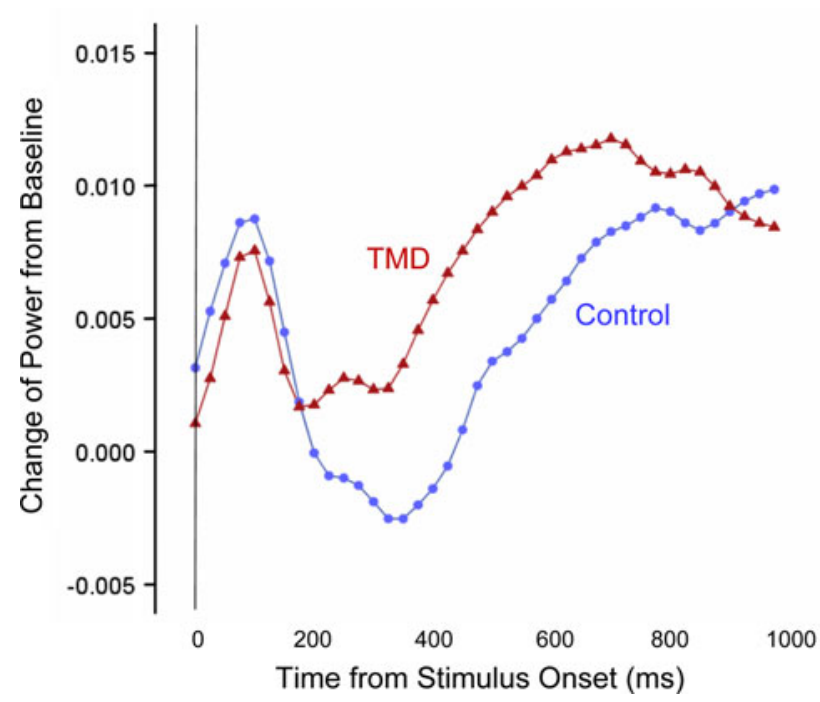

Fig. 13 Time course of power changes from baseline in the two groups for high frequencies $(21-160 \mathrm{~Hz})$

in the TMD pain group. A likely explanation for this finding is that the postulated cortical reorganization above has lowered the SII threshold for tactile stimuli.

There are several studies linking the areas above to tactile stimulation under various conditions, as follows. Temporally distinguishable responses to innocuous versus noxious stimuli have been reported in primate SI (Chen et al. 2004; Tommerdahl et al. 1996; Tommerdahl et al. 1998), and in human SI and SII (Chen et al. 2002). The participation of SII was reported in several functional imaging studies that involved light touch, pain perception, and visceral sensations (Aziz et al. 2000; Burton and Sinclair 2000; Coghill et al. 1994; Disbrow et al. 2000; Lam et al. 2001; Lotze et al. 2001; Peyron et al. 1999). SII responses to tactile stimulation may occur without task relevance (Disbrow et al. 2000; Eickhoff et al. 2006, 2007; Ruben et al. 2001). Insula is known to be an integration region for temperature, hunger, thirst, air hunger, and pain (Craig 2002; Frot et al. 2007). The insular cortex participates in acute and inflammatory nociception, and it has been involved in the descending pain modulation system (Burkey et al. 1996, 1999; Jasmin et al. 2003). With respect to the trigeminal nerve, Alvarez et al. (2009) demonstrated that the process of tactile stimulus can be changed in the insular cortex after trigeminal nerve injury in rats (Alvarez et al. 2009).

The results of the multivariate analyses above document various changes in the internal organization of the brain areas activated by the task in the two groups. These changes involve both a less tight association in the pain group (compared to the control group) of the areas related to the stimulus processing (Figs. 5, 6, MDS), as well as a differential composition of their internal clustering (Figs. 7 and 8, HTC). It is interesting that these changes are seen 
within a pattern that is overall very similar in the two groups, as reflected in the general distributions in MDS and the general tree structure in HTC. These findings lend credence to the hypothesis that the application of the stimulus elicits a specific cortical activation pattern, which is altered in its finer structure in the TMD pain group in a way suggesting a less coordinated information processing.

Finally, the results of the time-frequency analysis provided additional information on the dynamic aspects of the altered somatosensory brain processing mechanisms in the patients with TMD. Time-frequency analyses have been applied in a variety of somatosensory experiments (e.g., Ihara et al. 2003; Bauer et al. 2006; Hauck et al. 2007; Hsiao et al. 2008) where it yielded useful results. In this study, we found significant frequency-dependent differences in the changes from baseline between the two groups. These changes were stronger in the TMD group and were in opposite directions depending on the frequency, being more negative in the low $(4-20 \mathrm{~Hz})$ frequencies and more positive in higher $(21-160 \mathrm{~Hz})$ frequencies. This finding indicates a shift to higher frequency oscillations, at the expense of low frequency ones. Finally, these changes and group differences were consistent along time, throughout the $1 \mathrm{~s}$ from stimulus onset.

In summary, the results of the ECD and time-frequency analysis provide strong evidence for an altered brain function in TMD elicited by an innocuous tactile stimulation above the masseter muscle. This alteration was most prominent in dynamic aspects of brain function, notably onset time of brain activation (earlier in TMD), duration of activation (longer in TMD), functional grouping of key brain areas (less tight in TMD), and frequency-dependent, timesystematic changes of power from baseline (negative in low frequencies, positive in high frequencies in TMD). Altogether, these results make this simple innocuous stimulation attractive for possible wider application in evaluating brain function in TMD.

Acknowledgments We thank Dr. Eric Schiffman and Dr. Donald Nixdorf, Department of Diagnostic and Biological Sciences-TMD and Orofacial Pain Division, for helping selecting the TMD subjects. This work was supported by a Grant-In-Aid Program, Graduate School, University of Minnesota, to I. G. Koutlas, the U. S. Department of Veterans Affairs, and the American Legion Brain Sciences Chair.

\section{References}

Albuquerque RJ, de Leeuw R, Carlson CR, Okeson JP, Miller CS, Andersen AH (2006) Cerebral activation during thermal stimulation of patients who have burning mouth disorder: an fMRI study. Pain 122:223-234

Alvarez P, Dieb W, Hafidi A, Voisin DL, Dallel R (2009) Insular cortex representation of dynamic mechanical allodynia in trigeminal neuropathic rats. Neurobiol Dis 33:89-95
Apkarian AV, Bushnell MC, Treede RD, Zubieta JK (2005) Human brain mechanisms of pain perception and regulation in health and disease. Eur J Pain 9:463-484

Aziz Q, Schnitzler A, Enck P (2000) Functional neuroimaging of visceral sensation. J Clin Neurophysiol 17:604-612

Bauer M, Oostenveld R, Peeters M, Fries P (2006) Tactile spatial attention enhances gamma-band activity in somatosensory cortex and reduces low-frequency activity in parieto-occipital areas. J Neurosci 26:490-501

Bessho H, Shibukawa Y, Shintani M, Yajima Y, Suzuki T, Shibahara $\mathrm{T}$ (2007) Localization of palatal area in human somatosensory cortex. J Dent Res 86:265-270

Biermann K, Schmitz F, Witte OW, Konczak J, Freund HJ, Schnitzler A (1998) Interaction of finger representation in the human first somatosensory cortex: a neuromagnetic study. Neurosci Lett 251:13-16

Blatow M, Nennig E, Sarpaczki E, Reinhardt J, Schlieter M, Herweh C, Rasche D, Tronnier VM, Sartor K, Stippich C (2009) Altered somatosensory processing in trigeminal neuralgia. Hum Brain Mapp 30:3495-3508

Borsook D, DaSilva AF, Ploghaus A, Becerra L (2003) Specific and somatotopic functional magnetic resonance imaging activation in the trigeminal ganglion by brush and noxious heat. J Neurosci 23:7897-7903

Burkey AR, Carstens E, Wenniger JJ, Tang J, Jasmin L (1996) An opioidergic cortical antinociception triggering site in the agranular insular cortex of the rat that contributes to morphine antinociception. J Neurosci 16:6612-6623

Burkey AR, Carstens E, Jasmin L (1999) Dopamine reuptake inhibition in the rostral agranular insular cortex produces antinociception. J Neurosci 19:4169-4179

Burton H, Sinclair RJ (2000) Attending to and remembering tactile stimuli: a review of brain imaging data and single-neuron responses. J Clin Neurophysiol 17:575-591

Bushnell MC, Duncan GH, Hofbauer RK, Ha B, Chen JI, Carrier B (1999) Pain perception: is there a role for primary somatosensory cortex? Proc Natl Acad Sci U S A 96:7705-7709

Chen JI, Ha B, Bushnell MC, Pike B, Duncan GH (2002) Differentiating noxious- and innocuous-related activation of human somatosensory cortices using temporal analysis of fMRI. J Neurophysiol $88: 464-474$

Chen LM, Friedman RM, Roe AW (2004). Optical imaging reveals area-specific activation to thermal nociceptive stimuli in $\mathrm{S} 1$ of the squirrel monkey. Soc Neurosci Abstr

Coghill RC, Talbot JD, Evans AC, Meyer E, Gjedde A, Bushnell MC et al (1994) Distributed processing of pain and vibration by the human brain. J Neurosci 14:4095-4108

Cox DR, Oakes D (1984) Analysis of survival data. Chapman \& Hall/ CRC, Boca Raton

Craig AD (2002) How do you feel? Interoception: the sense of the physiological condition of the body. Nat Rev Neurosci 3:655666

DaSilva AF, Goadsby PJ, Borsook D (2007) Cluster headache: a review of neuroimaging findings. Curr Pain Headache Rep $11: 131-136$

DaSilva AF, Becerra L, Pendse G, Chizh B, Tully S, Borsook D (2008) Colocalized structural and functional changes in the cortex of patients with trigeminal neuropathic pain. PLoS ONE 3:e3396

de Leeuw R, Albuquerque R, Okeson J, Carlson C (2005) The contribution of neuroimaging techniques to the understanding of supraspinal pain circuits: implications for orofacial pain. Oral Surg Oral Med Oral Pathol Oral Radiol Endod 100:308-314

de Leeuw R, Davis CE, Albuquerque R, Carlson CR, Andersen AH (2006) Brain activity during stimulation of the trigeminal nerve with noxious heat. Oral Surg Oral Med Oral Pathol Oral Radiol Endod 102:750-757 
Derbyshire SW, Jones AK, Devani P, Friston KJ, Feinmann C, Harris $M$ et al (1994) Cerebral responses to pain in patients with atypical facial pain measured by positron emission tomography. J Neurol Neurosurg Psychiatry 57:1166-1172

Disbrow E, Roberts T, Krubitzer L (2000) Somatotopic organization of cortical fields in the lateral sulcus of Homo sapiens: evidence for SII and PV. J Comp Neurol 418:1-421

Dworkin SF, LeResche L (1992) Research diagnostic criteria for temporomandibular disorders: review, criteria, examinations and specifications, critique. J Craniomandib Disord 6:301355

Eickhoff SB, Lotze M, Wietek B, Amunts K, Enck P, Zilles K (2006) Segregation of visceral and somatosensory afferents: an fMRI and cytoarchitectonic mapping study. Neuroimage $31: 1004-1014$

Eickhoff SB, Grefkes C, Zilles K, Fink GR (2007). The somatotopic organization of cytoarchitectonic areas on the human parietal operculum. Cereb Cortex 171800-1811

Forss N, Jousmaki V (1998) Sensorimotor integration in human primary and secondary somatosensory cortices. Brain Res 781:259-267

Frot M, Magnin M, Mauguière F, Garcia-Larrea L (2007) Human SII and posterior insula differently encode thermal laser stimuli. Cereb Cortex 17:610-620

Hagelberg N, Forssell H, Rinne JO, Scheinin H, Taiminen T, Aalto S et al (2003) Striatal dopamine D1 and D2 receptors in burning mouth syndrome. Pain 101:149-154

Hamalainen M, Hari R, Ilmoniemi RJ, Knuutila J, Lounasmaa OV (1993) Magnetoencephalography: theory, instrumentation, and applications to noninvasive studies of working human brain. Rev Modern Phys 65:413-497

Hari R, Karhu J, Hamalainen M, Knuutila J, Salonen O, Sams M, Vilkman V (1993) Functional organization of the human first and second somatosensory cortices: a neuromagnetic study. Eur J Neurosci 5:724-734

Hauck M, Lorenz J, Engel AK (2007) Attention to painful stimulation enhances gamma-band activity and synchronization in human sensorimotor cortex. J Neurosci 27:9270-9277

Hoshiyama M, Kakigi R, Koyama S, Kitamura Y, Shimoio M, Watanabe S (1995) Somatosensory evoked magnetic fields after mechanical stimulation of the scalp in humans. Neurosci Lett 195:29-32

Hoshiyama M, Kakigi R, Koyama S, Kitamura Y, Shimoio M, Watanabe S (1996) Somatosensory evoked magnetic fields following stimulation of the lip in humans. Electroencephalogr Clin Neurophysiol 100:96-104

Hsiao FJ, Chen WT, Liao KK, Wu ZA, Ho LT, Lin YY (2008) Oscillatory characteristics of nociceptive responses in the SII cortex. Can J Neurol Sci 35:630-637

Ihara A, Hirata M, Yanagihara K, Ninomiya H, Imai K, Ishii R et al (2003) Neuromagnetic gamma-band activity in the primary and secondary somatosensory areas. Neuroreport 14:273-277

Inui K, Tran TD, Qiu Y, Wang X, Hoshiyama M, Kakigi R (2003) A comparative magnetoencephalographic study of cortical activations evoked by noxious and innocuous somatosensory stimulations. Neuroscience 120:235-248

Jantsch HH, Kemppainen P, Ringler R, Handwerker HO, Forster C (2005) Cortical representation of experimental tooth pain in humans. Pain 118:390-399

Jasmin L, Rabkin SD, Granato A, Boudah A, Ohara PT (2003) Analgesia and hyperalgesia from GABA-mediated modulation of the cerebral cortex. Nature 424:316-320

Jiang T, Li J, Jin Z, Wang YW, Feng HL, Ishikawa T (2006) Comparison of atypical orofacial pain and temporomandibular disorders synovitis pain processing in the human brain using functional magnetic resonance imaging. Zhonghua Kou Qiang Yi Xue Za Zhi 41:670-673
Jones EG (2000) Cortical and subcortical contributions to activitydependent plasticity in primate somatosensory cortex. Annu Rev Neurosci 23:1-37

Kakigi R, Koyama S, Hoshiyama M, Kitamura Y, Shimojo M, Watanabe $\mathrm{S}$ (1995) Pain-related magnetic fields following painful CO2 laser stimulation in man. Neurosci Lett 192:45-48

Kupers RC, Gybels JM, Gjedde A (2000) Positron emission tomography study of a chronic pain patient successfully treated with somatosensory thalamic stimulation. Pain 87:295-302

Kupers RC, Svensson P, Jensen TS (2004) Central representation of muscle pain and mechanical hyperesthesia in the orofacial region: a positron emission tomography study. Pain 108:284-293

Lam K, Kakigi R, Mukai T, Yamasaki H (2001) Attention and visual interference stimulation affect somatosensory processing: a magnetoencephalographic study. Neuroscience 104:689-703

Lang E, Kaltenhauser M, Seidler S, Mattenklodt P, Neundorfer B (2005) Persistent idiopathic facial pain exists independent of somatosensory input from the painful region: findings from quantitative sensory functions and somatotopy of the primary somatosensory cortex. Pain 118:80-91

Langheim FJ, Merkle AN, Leuthold AC, Lewis SM, Georgopoulos AP (2006) Dipole analysis of magnetoencephalographic data during continuous shape copying. Exp Brain Res 170:513-521

Leuthold AC (2003) Subtraction of heart artifact from MEG data: the matched filter revisited. Soc Neurosci Abs 863:15

Lotze M, Wietek B, Birbaumer N, Ehrhardt J, Grodd W, Enck P (2001) Cerebral activation during anal and rectal stimulation. Neuroimage 14:1027-1034

Maeda K, Kakigi R, Hoshiyama M, Koyama S (1999) Topography of the secondary somatosensory cortex in humans: a magnetoencephalo-graphic study. Neuroreport 10:301-306

Merchant H, Battaglia-Mayer A, Georgopoulos AP (2003) Functional organization of parietal neuronal responses to optic-flow stimuli. J Neurophysiol 90:675-682

Morelli N, Pesaresi I, Cafforio G, Maluccio MR, Gori S, Di Salle F, Murri L (2008) Functional magnetic resonance imaging in episodic cluster headache. J Headache Pain 10:11-14

Nagamatsu K, Nakasato N, Hatanaka K, Kanno A, Iwasaki M, Yoshimoto T (2001) Neuromagnetic localization of N15, the initial cortical response to lip stimulus. Neuroreport 12:1-5

Nakahara H, Nakasato N, Kanno A, Murayama S, Hatanaka K, Itoh H, Yoshimoto T (2004) Somatosensory-evoked fields for gingiva, lip, and tongue. J Dent Res 83:307-311

Nakamura A, Yamada T, Goto A, Kato T, Ito K, Abe Y, Kachi T, Kakigi R (1998) Somatosensory homunculus as drawn by MEG. Neuroimage 7:377-386

Nakasato N, Itoh H, Hatanaka K, Nakahara H, Kanno A, Yoshimoto T (2001) Movement-related magnetic fields to tongue protrusion. Neuroimage 14:924-935

Nash P, Henderson L, Macefield V, Klineberg I, Murray G (2007) Brain representation of noxious stimulation of masseter: an fMRI study. The IADR/AADR/CADR 85th General Session and Exhibition. Abstract 0259

Nevalainen P, Ramstad R, Isotalo E, Haapanen ML, Lauronen L (2006) Trigeminal somatosensory evoked magnetic fields to tactile stimulation. Clin Neurophysiol 117:2007-2015

Nguyen BT, Tran TD, Hoshiyama M, Inui K, Kakigi R (2004) Face representation in the human primary somatosensory cortex. Neurosci Res 50:227-232

Nguyen BT, Inui K, Hoshiyama M, Nakata H, Kakigi R (2005) Face representation in the human secondary somatosensory cortex. Clin Neurophysiol 116:1247-1253

Peyron R, Garcia-Larrea L, Gregoire MC, Costes N, Convers P, Lavenne $\mathrm{F}$ et al (1999) Haemodynamic brain responses to acute pain in humans: sensory and attentional networks. Brain $122: 1765-1780$ 
Ruben J, Schwiemann J, Deuchert M, Meyer R, Krause T, Curio G et al (2001) Somatotopic organization of human secondary somatosensory cortex. Cereb Cortex 11:463-473

Sakamoto K, Nakata H, Kakigi R (2008a) Somatotopic representation of the tongue in human secondary somatosensory cortex. Clin Neurophysiol 119:2125-2134

Sakamoto K, Nakata H, Kakigi R (2008b) Somatosensory-evoked magnetic fields following stimulation of the tongue in humans. Clin Neurophysiol 119:1664-1673

Shepard RN (1980) Multidimensional scaling, tree-fitting, and clustering. Science 210:390-398

Snedecor GW, Cochran WG (1989) Statistical methods. Iowa State University Press, Ames

Tagaris GA, Kim SG, Andersen P, Ugurbil K, Georgopoulos AP (1997) Mental rotation studied by functional magnetic resonance imaging at high field (4 Tesla): performance and cortical activation. J Cogn Neurosci 9:419-432

Tagaris GA, Richter W, Kim SG, Pellizzer G, Andersen P, Ugurbil K et al (1998) Functional magnetic resonance imaging of mental rotation and memory scanning: a multidimensional scaling analysis of brain activation patterns. Brain Res Brain Res Rev 26:106-112

Tailarach J, Tournoux P (1988) Co-planar stereotaxic atlas of the human brain. Thieme Medical Publishers, New York

Tommerdahl M, Delemos KA, Vierck CJ Jr, Favorov OV, Whitsel BL (1996) Anterior parietal cortical response to tactile and skin-heat- ing stimuli applied to the same skin site. J Neurophysiol 75:26622670

Tommerdahl M, Delemos KA, Favorov OV, Metz CB, Vierck CJ Jr, Whitsel BL (1998) Response of anterior parietal cortex to different modes of same-site skin stimulation. J Neurophysiol 80:32723283

Tronnier VM, Rasche D, Hamer J, Kienle AL, Kunze S (2001) Treatment of idiopathic trigeminal neuralgia: comparison of long-term outcome after radiofrequency rhizotomy and microvascular decompression. Neurosurgery 48:1261-1267

Tzagarakis C, Jerde TA, Lewis SM, Ugurbil K, Georgopoulos AP (2009) Cerebral cortical mechanisms of copying geometrical shapes: a multidimensional scaling analysis of fMRI patterns of activation. Exp Brain Res 194:369-380

Uutela K, Hamalainen M, Somersalo E (1999) Visualization of magnetoencephalographic data using minimum current estimates. Neuroimage 10:173-180

Wolters CH, Beckmann RF, Rienacker A, Buchner H (1999) Comparing regularized and non-regularized nonlinear dipole fit methods: a study in a simulated sulcus structure. Brain Topogr 12:3-18

Yang TT, Gallen CC, Schwartz BJ, Bloom FE (1993) Noninvasive somatosensory homunculus mapping in humans by using a largearray biomagnetometer. Proc Natl Acad Sci U S A 90:3098-3102 